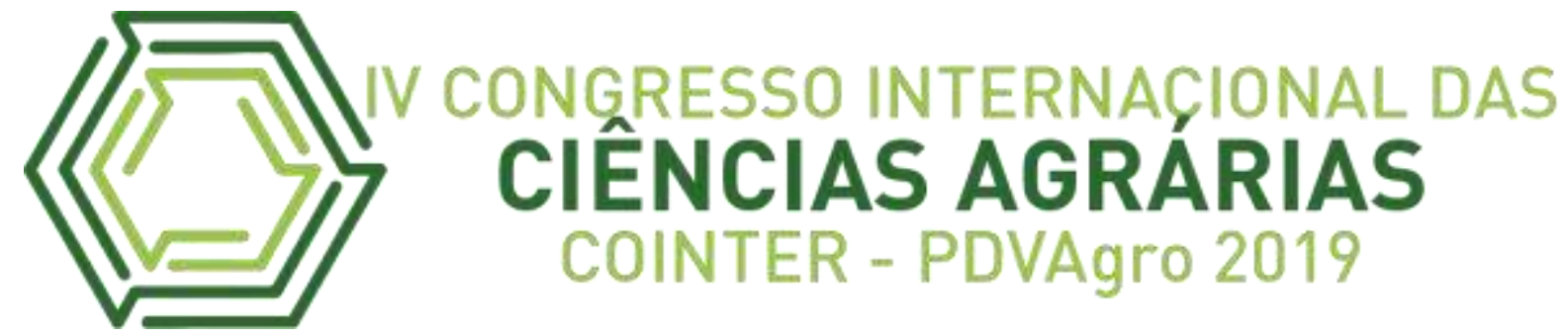

\title{
CINÉTICA DE DEGRADAÇÃO DO ÁCIDO ASCÓRBICO E DA COR EM POLPAS DE TAMARINDO CONGELADAS
}

\section{CINÉTICA DE ÁCIDO ASCÓRBICO Y DEGRADACIÓN DEL COLOR EN LA PULPA DE TAMARINA CONGELADA}

\section{KINETICS OF ASCORBIC ACID AND COLOR DEGRADATION IN FROZEN TAMARIN PULP}

\author{
Apresentação: Comunicação Oral
}

Sonara de França Sousa ${ }^{1}$; Krause Gonçalves Silveira Albuquerque ${ }^{2}$; Josivanda Palmeira Gomes $^{3}$

\section{DOI: https://doi.org/10.31692/2526-7701.IVCOINTERPDVAgro.2019.0156}

\begin{abstract}
Resumo
O conhecimento da cinética de degradação é suma importância para prever a perda de qualidade dos alimentos durante o armazenamento. Em vista disso, objetivou-se com o presente trabalho estudar a cinética de degradação do ácido ascórbico e da cor em polpas de tamarindo armazenadas a temperatura de congelamento comercial. As seguintes análises foram realizadas nas polpas integral e adicionadas de maltodextrina (10, 15 e 20\%): teor de água, ácido ascórbico e cor (tonalidade e índice de escurecimento), avaliadas a cada 15 dias por 75 dias. O delineamento experimental foi o inteiramente casualizado com esquema fatorial $6 \times 4$ (tempo $\mathrm{x}$ concentração). Durante o armazenamento congelado, as polpas apresentaram aumento nos teores de água, este fato pode ser atribuido à embalagem utilizada que não apresentou uma boa barreira à entrada de vapor d'água. Os teores de ácido ascórbico apresentaram decréscimos com tempo de armazenamento, e, de acordo com a meia-vida do produto, a polpa integral de tamarindo apresentou validade de 60 dias, já as polpas adicionadas de maltodextrina, a validade foi prorrogada por 75 dias. O modelo de Weibull apresentou um bom ajuste para descrever a cinética de degradação do ácido ascórbico, onde a velocidade de degradação foi maior na polpa integral, evidenciando que o coadjuvante utilizado exerceu um efeito protetor. Com relação à cor, as médias obtidas para a tonalidade revelaram que as amostras apresentaram ângulos próximos a $0^{\circ}$, evidenciando o vermelho. $\mathrm{O}$ índice de escurecimento diminuiu de acordo com a progressão do tempo e aumento da concentração de maltodextrina. A regressão linear utilizada para descrever a cinética e degradação da cor nas polpas mostrou-se satisfatória para fornecer a informações a respeito da velocidade de degradação.
\end{abstract}

\footnotetext{
${ }^{1}$ Doutora em Engenharia de Processos, Universidade Federal de Campina Grande, sonara_franca@yahoo.com.br

2 Graduando em Engenharia de Alimentos, Universidade Federal Rural de Pernambuco, albuquerque.k.g.s@gmail.com

${ }_{3}^{3}$ Professora do Programa de Pós-graduação em Engenharia de Processos, Universidade Federal de Campina Grande, josivanda@gmail.com
} 
Palavras-Chave: Tamarindus indica. L, modelo de Weibull, maltodextrina

\begin{abstract}
Resumen
El conocimiento de la cinética de degradación es de suma importancia para predecir la pérdida de calidad de los alimentos durante el almacenamiento. Por lo tanto, el objetivo de este trabajo fue estudiar la cinética de la degradación del ácido ascórbico y el color en las pulpas de tamarindo almacenadas a temperatura de congelación comercial. Los siguientes análisis se realizaron en las pulpas completas y se añadió maltodextrina (10, 15 y 20\%): contenido de agua, ácido ascórbico y color (índice de sombra y oscurecimiento), evaluado cada 15 días durante 75 días. El diseño experimental fue completamente al azar con un esquema factorial de $6 \times 4$ (tiempo x concentración). Durante el almacenamiento congelado, las pulpas presentaron un aumento en el contenido de agua, este hecho puede atribuirse al embalaje usado que no presentaba una buena barrera para la entrada de vapor de agua. Los niveles de ácido ascórbico disminuyeron con el tiempo de almacenamiento y, de acuerdo con la vida media del producto, la pulpa entera de tamarindo fue válida durante 60 días, mientras que la pulpa con maltodextrina añadida se extendió durante 75 días. El modelo de Weibull presentó un buen ajuste para describir la cinética de degradación del ácido ascórbico, donde la velocidad de degradación fue mayor en la pulpa integral, lo que demuestra que el adyuvante utilizado tenía un efecto protector. Con respecto al color, los promedios obtenidos para el tono revelaron que las muestras tenían ángulos cercanos a $0^{\circ}$, mostrando rojo. El índice de oscurecimiento disminuyó con la progresión del tiempo y el aumento de la concentración de maltodextrina. La regresión lineal utilizada para describir la cinética y la degradación del color en las pulpas fue satisfactoria para proporcionar información sobre la tasa de degradación.
\end{abstract}

Palabras Clave: Tamarindus indica. L, modelo de Weibull, maltodextrina

\begin{abstract}
The knowledge of the degradation kinetics is of paramount importance to predict the loss of food quality during storage. Therefore, the objective of the present work was to study the kinetics of ascorbic acid degradation and color in tamarind pulps stored at commercial freezing temperature. The following analyzes were performed on whole pulps and added maltodextrin $(10,15$ and 20\%): water content, ascorbic acid and color (hue and darkening index), evaluated every 15 days for 75 days. The experimental design was completely randomized with a $6 \times 4$ factorial scheme (time $x$ concentration). During frozen storage, the pulps presented an increase in water contents, this fact can be attributed to the used packaging that did not present a good barrier to the entrance of water vapor. Ascorbic acid levels decreased with storage time, and, according to the half-life of the product, the tamarind whole pulp was valid for 60 days, while the maltodextrin added pulp was extended for 75 days. The Weibull model presented a good fit to describe the degradation kinetics of ascorbic acid, where the degradation velocity was higher in the integral pulp, showing that the adjuvant used had a protective effect. Regarding the color, the averages obtained for the shade revealed that the samples had angles close to $0^{\circ}$, showing red. The darkening index decreased as time progressed and the maltodextrin concentration increased. The linear regression used to describe the kinetics and color degradation in the pulps
\end{abstract}


was satisfactory to provide information about the degradation rate.

Keywords: Tamarindus indica. L, Weibull model, maltodextrin

\section{Introdução}

O Cerrado, segundo maior bioma do Brasil, apresenta uma rica biodiversidade vegetal que possui elevado potencial econômico, nutricional e tecnológico. Entre as espécies frutíferas presentes destaca-se o tamarindo (Tamarindus indica L.), nativa da África Tropical, pertencente à família Fabaceae, e frequentemente encontrada em regiões de clima tropical e subtropical no país (HAMACEK et al. 2011).

A comercialização dos frutos pelos agricultores familiares é geralmente realizada em feiras livres, no entanto, por serem de casca frágil e sujeito a deterioração fungica, os tamarindos têm sua comercialização in natura dificultada a grandes distâncias. Neste contexto, a produção de polpas de frutas congeladas torna-se um meio favorável para o aproveitamento integral das frutas na época da safra evitando os problemas ligados a sazonalidade. Para que se produza uma polpa de fruta congelada com qualidade é preciso atentar-se à manutenção dos nutrientes e às suas características físico-químicas, microbiológicas e sensoriais, que devem estar próximas as da fruta in natura, de forma a satisfazer as exigências do consumidor e da legislação vigente (DANTAS et al., 2012).

O ácido ascórbico está amplamente distribuído nos produtos de origem vegetal, e é considerado hidrossolúvel e termolábil. Sabe-se que diversos fatores afetam a sua estabilidade durante o processamento e armazenamento, incluindo o $\mathrm{pH}$ do meio, a presença de oxigênio e de íons metálicos, e a temperatura (TARRAGO-TRANI et al., 2012; SPINOLA et al., 2013). A cor, por sua vez, é o atributo de qualidade mais atrativo para o consumidor, pois está associada com a maturação, frescor e também ao sabor, sendo considerada o parâmetro que, em primeiro lugar, atinge o olhar do comprador. A degradação da cor do material pode ser avaliada com base no índice de escurecimento (IE), calculado a partir das coordenadas $\mathrm{L} *$, a * e b * do sistema Hunter (TREVISAN et al., 2006; PONKHAM et al., 2012).

O conhecimento da cinética de degradação, incluindo a ordem de reação, constantes de velocidade e a energia de ativação, é muito importante para prever a perda de qualidade dos alimentos durante o armazenamento (PATRAS et al., 2010). O uso de modelos matemáticos cinéticos facilita consideravelmente a otimização, o design, simulação e controle de projetos 
industriais e contribui para uma melhor utilização do tempo e energia (SANT'ANNA et al., 2012).

Trabalhos relacionados à degradação do ácido ascórbico durante o processamento de alimentos vegetais já foram registrados em várias investigações (FERNÁNDEZ et al., 2011; GEORGÉ et al., 2011; MERCALI et al., 2012; CUNHA t al., 2014), bem como a degradação da cor (MOURA et al., 2001; AQUINO et al., 2011; MATSUDA et al., 2013). No entanto, informações sobre a degradação durante o congelamento da polpa de tamarindo são incipientes.

Diante do exposto, objetivou-se com o presente trabalho estudar a cinética de degradação do ácido ascórbico e da cor em polpas de tamarindo armazenadas na temperatura de $-18^{\circ} \mathrm{C}$, por um período de 75 dias.

\section{Fundamentação Teórica}

A vida-de-prateleira dos alimentos pode ser influenciada por diversos fatores, tais como as condições de processamento, tipo de embalagem, carga microbiana inicial, tempo e temperatura de armazenamento. Dentre estes fatores, a temperatura é geralmente o mais determinante, pois pode acelerar a oxidação de certos nutrientes, e alterar as propriedades nutritivas e sensoriais dos produtos (OLIVEIRA et al., 2013).

Na tecnologia de alimentos, busca-se a maximização da retenção dos nutrientes durante o processamento e o armazenamento dos produtos. O ácido ascórbico é um importante indicador, pois sendo a vitamina mais termolábil, sua presença no alimento indica que, provavelmente, os demais nutrientes também estão sendo preservados (ALVES et al., 2010). Por isso, o ácido ascórbico é considerado como indicativo de qualidade dos nutrientes durante o processamento e o armazenamento de alimentos. A cinética de degradação do ácido ascórbico pode ser descrita pela equação de Weibull, usada para descrever sistemas ou eventos que tenham algum grau de variabilidade. O modelo de Weibull também já foi usado para descrever o crescimento microbiano, a inativação microbiana e a degradação de nutrientes e enzimas (DEROSSI et al., 2010).

A cor é um atributo de grande importância para a indústria de alimentos, visto que é um parâmetro de qualidade capaz de influenciar a aceitação dos produtos alimentícios. Na medição de cor em alimentos, o sistema $\mathrm{L}^{*} \mathrm{a}^{*} \mathrm{~b}^{*}$ é o mais utilizado devido a uma distribuição uniforme de cores, e porque a distância entre duas cores diferentes corresponde, aproximadamente, à diferença de cor percebida pelo olho humano (WU e SUN, 2013). A avaliação das alterações 
de cor nos alimentos é realizada por aparelhos específicos que ao iluminar a amostra medem a energia luminosa transmitida ou refletida pela superfície, como em um espectrofotômetro e em um colorímetro tristímulo, os quais descrevem a deterioração visual da cor (FANTE, 2011).

A cinética de degradação da cor em alimentos é complexa. Modelos seguros que predizem corretamente o processo da reação química são proveitosos em muitas aplicações da engenharia incluindo processos de otimização. Estudos experimentais e aplicação de modelos para predizer e interpretar parâmetros cinéticos como a ordem de reação são necessários (AHMED et al., 2002).

\section{Metodologia}

Local do Experimento

O trabalho foi desenvolvido no Laboratório de Armazenamento e Processamento de Produtos Agrícolas (LAPPA), da Unidade Acadêmica de Engenharia Agrícola da Universidade Federal de Campina Grande, Campina Grande (PB).

Aquisição e Processamento da matéria-prima

Foram utilizados como matéria-prima tamarindos, safra 2017, adquiridos na zona rural do município de Juazeiro do Norte (CE). Os frutos foram selecionados de acordo com o grau de maturação, onde os mesmos apresentaram casca quebradiça e a polpa marrom-escura, adequados para o consumo e processamento. Em seguida, realizou-se a lavagem em água corrente e a sanitização por imersão em solução de hipoclorito de sódio (30ppm) por 5 minutos, seguido por um enxague com água potável, drenando-se o excesso de água dos frutos e procedendo-se a separação manual da casca.

Os tamarindos descascados foram imersos em água destilada por 20 minutos, na proporção 1:1, para facilitar o desprendimento da polpa das sementes, sendo despolpados em despolpadeira (Laboremos DF 200). A polpa obtida foi homogeneizada com três formulações de maltodextrina (DE 10) nas concentrações de 10, 15 e 20\%, definidas por pré-testes. A amostra controle e as formuladas foram envasadas em embalagens plásticas transparentes de polietileno de baixa densidade, com capacidade para $250 \mathrm{~g}$ de polpa, sendo posteriormente termosseladas. 
$\mathrm{O}$ armazenamento foi feito em freezer horizontal na temperatura fixa de $-18{ }^{\circ} \mathrm{C}$, usualmente utilizada na comercialização, durante 75 dias, sendo analisadas a cada 15 dias, a partir do tempo 0 .

Parâmetros analisados

O teor de ácido ascórbico foi determinado por titulometria com 2,6diclorofenolindofenol de sódio padronizado da qual, utiliza o ácido oxálico como solução extratora, os resultados foram expressos em mg de ácido ascórbico por $100 \mathrm{~g}^{-1}$ da amostra, conforme a metodologia da AOAC (2000); e a cor foi determinada por leitura direta, utilizando o espectofotômetro MiniScan HunterLab XE Plus, equipado com iluminante D65, ângulo de observação de $10^{\circ}$ e calibrado com placa padrão branca e preta $(X=80,5 ; Y=85,3 ; Z=90,0)$ A partir das coordenadas $L^{*}$ (luminosidade), $+a^{*}$ (intensidade vermelho) $\mathrm{e}+\mathrm{b}^{*}$ (intensidade amarelo) obtidas nas amostras, foram calculadas as coordenadas $\mathrm{h}^{\circ}$ (tonalidade) e índice de escurecimento (IE), de acordo com as Equações 01 e 02, respectivamente.

$$
\begin{aligned}
& h^{*}=\tan ^{-1}\left(\frac{b^{*}}{a^{*}}\right) \\
& I E=\frac{[100(X-0,031)]}{0,172}
\end{aligned}
$$

Em que: $X=\frac{a^{*}+1,75 L^{*}}{\left(5,645 L^{*}+a^{*}-3,02 b^{*}\right)}$

Modelos da cinética de degradação do ácido ascórbico

Utilizou-se o modelo de Weibull, representado pela Equação 03:

$$
\frac{C}{C o}=\exp \left(-k * t^{\alpha}\right)
$$

Onde: Co e C representam a concentração de ácido ascórbico inicial e final, respectivamente; $\mathrm{k}$ é a velocidade da constante de primeira ordem $\left(\min ^{-1}\right) ; \alpha$ é a é o fator de forma (adimensional); e t é o tempo de armazenamento (dias).

Modelos cinéticos de degradação da cor 
Para a determinação da ordem de reação e sua constante de velocidade, foram plotados os valores de tonalidade e índice de escurecimento versus o tempo (dias) de armazenamento para cada amostra, e realizada a regressão linear destes valores. A inclinação obtida na regressão linear corresponde aos valores de k (velocidade de reação).

\section{Análise estatística}

Para a análise dos fatores quantitativos, foi realizada a regressão linear em que houve efeito significativo do tempo. As constantes das cinéticas de degradação e a plotagem dos gráficos foram obtidas com o auxílio dos programas estatísticos STATISTIC 7 e ORIGINPro 8.

\section{Resultados e Discussão}

Na Figura 1 encontram-se os resultados da degradação do ácido ascórbico das polpas de tamarindo integral e formuladas durante o armazenamento a $-18^{\circ} \mathrm{C}$.

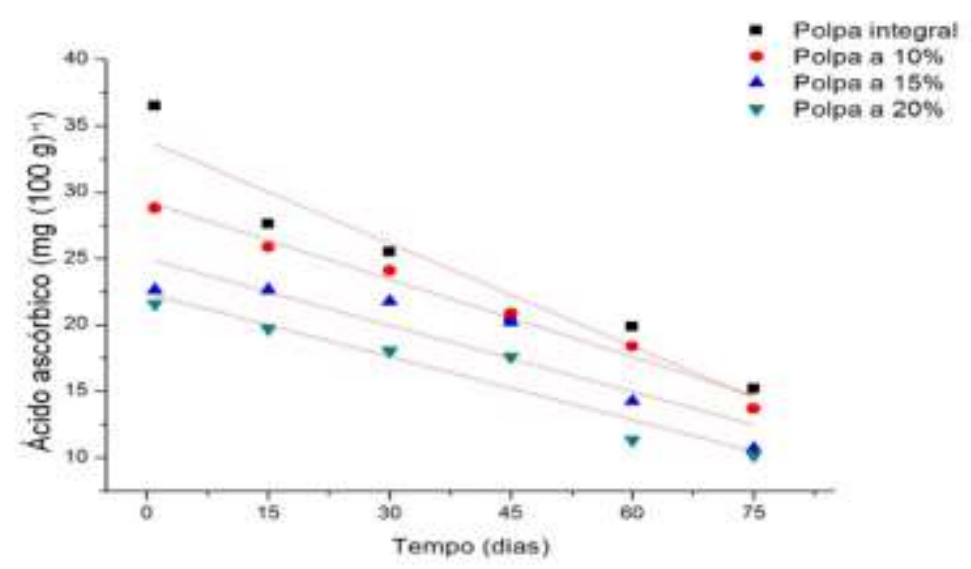

Figura 1 - Relação do teor de ácido ascórbico durante o tempo de armazenamento (dias). Fonte própria (2019)

A redução do teor desta vitamina foi de 58,36, 52,50, 52,83 e 52,81\% para as amostras a $0,10,15$ e 20\%, respectivamente, evidenciando que a adição de maltodextrina exerceu influencias significativas ao nível de 5\% de probabilidade. Na Tabela 1 encontra-se o ajuste da equação linear aos dados experimentais. Percebe-se que os valores de $\mathrm{R}^{2} \geq 0,850$, considerado satisfatórios, podendo ser utilizada para predizer o comportamento das amostras durante o tempo de armazenamento. 
Tabela 1 - Equação linear e coeficiente de determinação para as polpas de tamarindo integral e formuladas com maltodextrina

\begin{tabular}{ccc}
\hline Polpa (\%) & Equação & $\mathbf{R}^{2}$ \\
\hline 0 & $\mathrm{y}=-0,2587 \mathrm{x}+33,91$ & 0,925 \\
10 & $\mathrm{y}=-0,1949 \mathrm{x}+29,307$ & 0,983 \\
15 & $\mathrm{y}=-0,1671 \mathrm{x}+24,997$ & 0,850 \\
20 & $\mathrm{y}=-0,1589 \mathrm{x}+22,394$ & 0,919 \\
\hline
\end{tabular}

Fonte própria (2019)

Santos et al. (2014), afirmam que as taxas de degradação dos ácidos orgânicos, particularmente da vitamina $\mathrm{C}$ em frutas, são afetados por fatores como, temperatura, umidade e tempo de armazenamento. Com isso, as perdas da qualidade nutricional e do frescor das polpas de frutas congeladas podem ser avaliadas com base na redução dos teores dos ácidos orgânicos durante o período de estocagem.

Segundo a ANVISA (2017), a data de vencimento da qualidade do produto alimentício será indicada pelo próprio fabricante, mas, para isso, este fica obrigado a apresentar um estudo de estabilidade de seus produtos. De acordo com as taxas de degradação apresentadas, o período de validade da polpa de fruta pode ser obtido com base na cinética de degradação do ácido ascórbico. Considerando-se como concentração mínima aceitável o valor equivalente a $50 \%$ do teor inicial do ácido encontrado no produto (meia-vida), o prazo de validade remanescente do produto pode ser estimado em 60 dias para a polpa integral, e 75 dias para as polpas adicionadas de maltodextrina. Deve-se destacar que o prazo de validade é o tempo médio que um produto não apresenta deterioração, desde que respeitadas às condições de armazenamento indicadas na embalagem. Na Tabela 2 encontram-se as constantes e os coeficientes de determinação da Equação cinética de Weibull.

Tabela 2- Parâmetros e coeficientes de determinação da equação cinética de Weibull aplicada às polpas 
de tamarindo

\begin{tabular}{cccc}
\hline $\begin{array}{c}\text { Polpa } \\
(\%)\end{array}$ & k $\left(\right.$ horas $\left.^{-1}\right)$ & $\boldsymbol{\alpha}$ & $\mathbf{R}^{\mathbf{2}}$ \\
\hline 0 & $2,52 \times 10^{-3}$ & 0,771 & 0,973 \\
10 & $2,67 \times 10^{-4}$ & 1,009 & 0,908 \\
15 & $2,41 \times 10^{-4}$ & 1,089 & 0,848 \\
20 & $2,39 \times 10^{-4}$ & 1,027 & 0,827 \\
\hline
\end{tabular}

Fonte própria (2019)

Os valores de k referem-se à velocidade de degradação do ácido ascórbico, observa-se que esta velocidade é maior na polpa integral de tamarindo, ao passo que, as polpas adicionadas de maltodextrina apresentam uma menor degradação, supõe-se que o coadjuvante utilizado exerça um efeito protetor sobre este nutriente, no entanto, pesquisas que comprovem este resultado não foram encontradas na literatura.

A degradação provavelmente deve-se às reações químicas envolvendo oxidação e formação de ácido desidroascórbico (DHAA), hidrólise do DHAA em ácido 2,3-dicicogulônico e a geração, por polimerização, de produtos inativos nutricionalmente (DEWANTO et al., 2002). As constantes cinéticas podem ser observadas em diversos trabalhos, a saber, OrdónezSantos et al. (2013), ao estudarem a cinética de degradação do ácido ascórbico em goiabas nas temperaturas de 75,85 e $95{ }^{\circ} \mathrm{C}$ por $10,20,30$ e 40 minutos, onde os valores de $\mathrm{k}$ foram 0,80 , 1,12 e 1,45 $\left(\mathrm{min}^{-1}\right)$, respectivamente. Oliveira et al. (2013), ao estudarem a cinética de degradação do suco integral de manga $\left(25,35\right.$ e $\left.45^{\circ} \mathrm{C}\right)$ durante 120 dias, onde as constantes foram de 0,0039, 0,0080 e 0,0161 (dias $\left.{ }^{-1}\right)$, respectivamente, e Paul e Ghosh (2012), registrando em sucos de romã $\left(70\right.$ a $90^{\circ} \mathrm{C}$ por 90 minuto) valores de $\mathrm{k}$ de 0,00288 a $0,00382\left(\mathrm{~min}^{-1}\right)$.

Ainda de acordo com a Tabela 2, nota-se que $\alpha$ foi menor que 1 para a polpa integral $(0,771)$ e superiores à unidade nas polpas adicionadas de maltodextrina. Quando $\alpha$ apresenta valor igual à unidade, significa que o modelo de Weibull representa uma cinética de $1^{\mathrm{a}}$ ordem. A maioria dos trabalhos na literatura utiliza a cinética de primeira ordem para descrever a degradação do ácido ascórbico (NISHA et al., 2004; CASTRO et al., 2004; ALVES et al. 2010; HAL et al., 2012). O modelo de Weibull é usado para descrever sistemas ou eventos que tenham algum grau de variabilidade. Este modelo já foi usado para descrever o crescimento e inativação microbiana e a degradação de nutrientes e enzimas (DEROSSI et al., 2010). Segundo os 
mesmos autores, em termos de degradação do ácido ascórbico, este modelo é utilizado para descrever tal comportamento em vegetais congelados.

A Figura 2 apresenta o ajuste do modelo para as polpas de tamarindo integral e formuladas. $\mathrm{O}$ ajuste aos dados foi considerado satisfatório, em especial, para a polpa integral e a $10 \%$, onde os valores de $\mathrm{R}^{2}$ foram 0,973 e 0,908 , respectivamente.

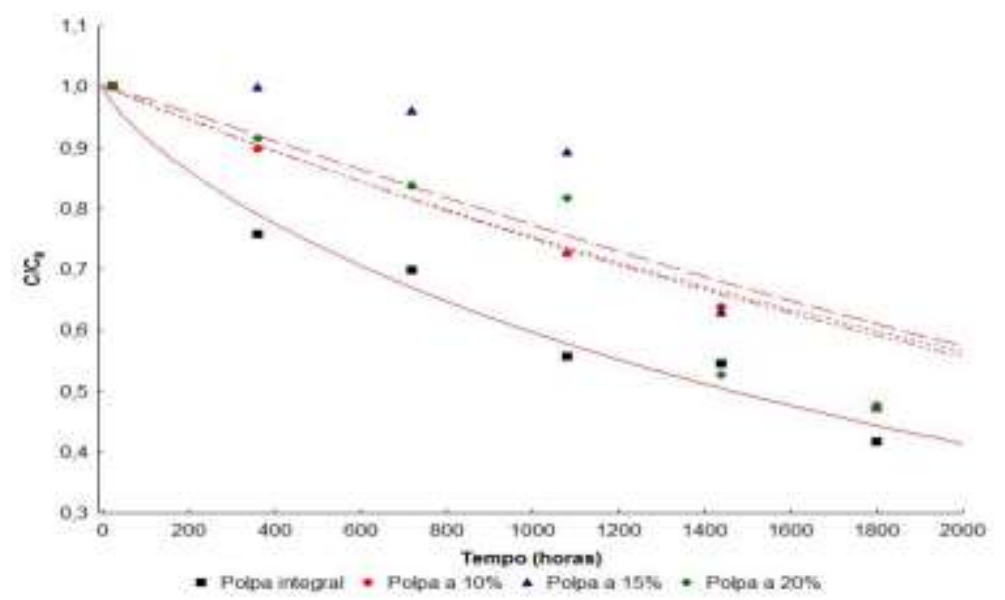

Figura 2 - Ajuste dos dados de degradação do ácido ascórbico de polpa de tamarindo integral e formulada com 10, 15 e 20\% de maltodextrina ao modelo de Weibull. Fonte própria (2019)

$\mathrm{Na}$ Figura 3 tem-se as médias para a tonalidade das polpas integral e formuladas. Ângulos de $0^{\circ}$ ou $360^{\circ}$ representam tonalidade vermelha, enquanto que os ângulos $90^{\circ}, 180^{\circ} \mathrm{e}$ $127^{\circ}$ representam amarelo, verde e azul, respectivamente (PATHARE et al., 2013). Percebe-se que as médias alcançadas apresentam valores próximos a $0^{\circ}$, indicando uma polpa de coloração vermelha, que se torna mais intensa com a progressão do armazenamento.

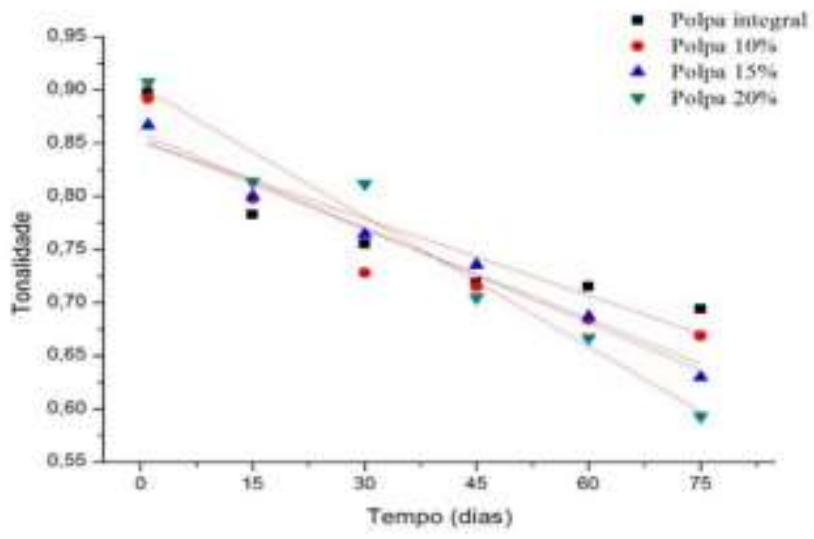

Figura 3- Ângulo de tonalidade das polpas de tamarindo integral e formuladas com 10, 15 e $20 \%$ de 
maltodextrina durante o armazenamento congelado. Fonte própria (2019)

Na Tabela 3, são apresentados os valores de $\mathrm{k}$ e os coeficientes de determinação da equação linear aplicada aos dados da tonalidade das amostras, podemos observar que a velocidade da degradação aumenta no sentido da maior concentração do coadjuvante utilizado, ou seja, a tonalidade diminui e isso pode ser explicado devido à maltodextrina diminuir os valores do ângulo Hue, reduzindo sua intensidade. Os valores de $\mathrm{R}^{2}$ mostraram-se satisfatórios para as amostras a 15 e $20 \%$ de maltodextrina, a saber, 0,961 e 0,922, respectivamente.

Tabela 3 - Parâmetros k e coeficientes de determinação da regressão linear aplicada à tonalidade das polpas de tamarindo armazenadas.

\begin{tabular}{ccc}
\hline Polpa (\%) & $\mathbf{k}\left(\mathbf{d i a s}^{-\mathbf{1}}\right)$ & $\mathbf{R}^{\mathbf{2}}$ \\
\hline 0 & 0,0024 & 0,585 \\
10 & 0,0028 & 0,711 \\
15 & 0,0030 & 0,961 \\
20 & 0,0041 & 0,922
\end{tabular}

Fonte própria (2019)

Na Figura 4 encontra-se o gráfico da regressão linear referente aos valores do índice de escurecimento, observa-se que os pontos apresentam-se bem distintos para todas as amostras 
estudadas e que os índices de escurecimento diminuíram significativamente de acordo com o aumento das variáveis tempo e concentração de maltodextrina.

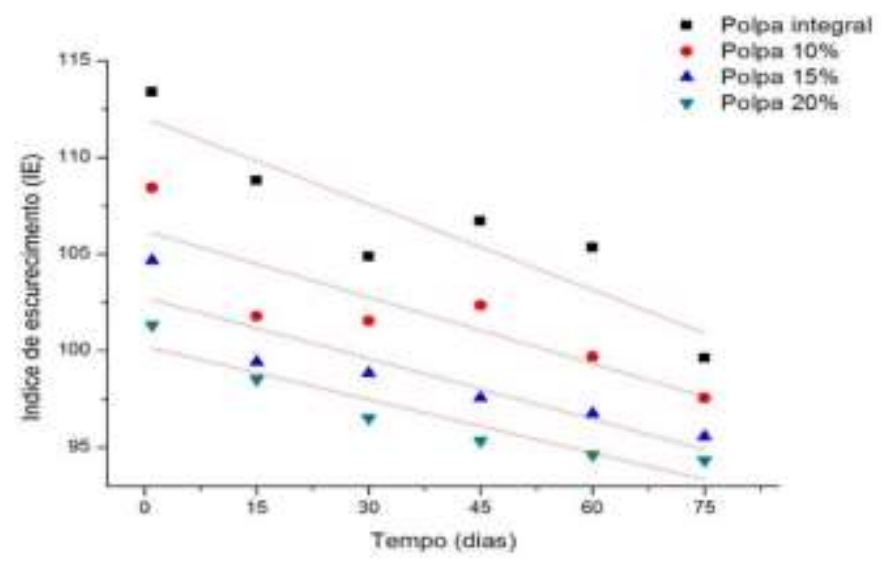

Figura 4 - Regressão não linear dos valores do índice de escurecimento nas polpas de tamarindo. Fonte própria (2019)

O índice de escurecimento mede a pureza da cor castanha na amostra e por isso é um parâmetro que indica a formação dos pigmentos amarronzados decorrente das reações de escurecimento, como a oxidação da vitamina C, que ocasionam a formação de pigmentos escuros no produto (BOREL et al., 2015).

$\mathrm{Na}$ Tabela 4 são apresentadas as constantes $\mathrm{k}$ e seus respectivos coeficientes de determinação para o índice de escurecimento. A velocidade das reações diminuiu com o aumento das concentrações de maltodextrina, indicando que a formação de pigmentos escuros é menor nestas condições e que o coadjuvante utilizado age camuflando o escurecimento das amostras.

Tabela 4 - Parâmetros e coeficientes de determinação da regressão não linear aplicada ao índice de 
escurecimento das popas de tamarindo

\begin{tabular}{ccc}
\hline Polpa (\%) & $\mathbf{k}\left(\right.$ dias $\left.^{-1}\right)$ & $\mathbf{R}^{\mathbf{2}}$ \\
\hline 0 & 0,1484 & 0,5984 \\
10 & 0,1147 & 0,4879 \\
15 & 0,1050 & 0,6255 \\
20 & 0,0919 & 0,7446 \\
\hline
\end{tabular}

Fonte própria (2019)

Dados sobre as constantes cinéticas do índice de escurecimento em polpas de frutas congeladas são escassos, o resultado obtido pode ser comparado com outros parâmetros de coloração. Oliveira et al. (2013), obtiveram constantes cinéticas de 0,0187 e 0,0487 dias ${ }^{-1}$ referente a luminosidade do suco de manga armazenados a 35 e $45^{\circ} \mathrm{C}$, respectivamente, e 0,0313 e 0,110 dias $^{-1}$ referente a diferença total e cor das amostras armazenadas sob estas mesmas temperaturas.

\section{Conclusões}

No armazenamento congelado, os teores de ácido ascórbico apresentam decréscimos com tempo de armazenamento, a polpa integral de tamarindo apresenta validade de 60 dias, enquanto que as polpas adicionadas de maltodextrina apresentam validade de 75 dias de acordo com a meia vida deste nutriente.

O modelo de Weibull apresenta um bom ajuste para descrever a cinética de degradação do ácido ascórbico, sendo verificado que a velocidade de degradação é maior na polpa integral, evidenciando que o coadjuvante utilizado exerce um efeito protetor sobre o ácido ascórbico.

Com relação à cor, as médias obtidas para o parâmetro tonalidade revelam que as amostras apresentam ângulos próximos a $0^{\circ}$, evidenciando o vermelho. $O$ índice de escurecimento diminui de acordo com o aumento das variáveis, tempo e concentração de maltodextrina. A regressão linear utilizada para descrever a cinética e degradação da cor nas polpas é eficiente para fornecer a informações à respeito da velocidade de degradação.

\section{Referências}

AHMED, J.; SHIVHARE, U. S.; SANDHU, S. Thermal degradation kinetics of carotenoids and visual color of papaya puree. J. Food Sci., v. 67, p. 2692-2695, 2002. 
ALVES, J.A.; NASSUR, R.C.M.R; PIRES, C.R.F; ALCÂNTARA, E.M.; GIANNONI, J.A.; LIMA, L.C.O. Cinética de degradação de vitamina C em mangas "Palmer" minimamente processadas armazenadas em diferentes temperaturas. Ciência e Agrotecnologia, v. 34, n. 3, p. 714-721, 2010.

ANVISA - Agência Nacional de Vigilância Sanitária. Resolução CISA/MA/MS no 10, de 31 de julho de 1984. Disponível em: http://www.anvisa.gov.br/anvisalegis/resol/10_84.htm. Acessado em: 04 de Sete. 2017

AOAC - Associaton of Official Analytical Chemists. Official Methods of Analysis of AOAC International. v.2. 17.ed. Arlington, 2000.

AQUINO, A. C. M. S.; MOÉS, R. S.; CASTRO, A. A. Estabilidade de ácido ascórbico, carotenoides e antocianinas de frutos de acerola congelados por métodos criogênicos. Brazilian Journal Food and Technology, v. 14, n. 2, p. 154-163, 2011.

BOREL, L. D. M.; LINHARES, M. C.; PRADO, M.M. Cinética de degradação da cor de pólen apícola durante a secagem infravermelho. In: Congresso Brasileiro de Sistemas Particulados, 37, 2015. São Carlos (SP). Anais... Universidade Federal de São Carlos, 2015. p 1-10.

CASTRO, I.; TEIXEIRA, J.A.; SALENGKE, S.; SASTRY, S.K.; VICENTE, A.A. Ohmic heating of strawberry products: electrical conductivity measurements and ascorbic acid degradation kinetics. Innovative Food Science \& Emerging Technologies, v.5, n.1, p.27- 36, 2004.

CUNHA, K.D.; SILVA, P. R.; COSTA, L. F. S. F.; TEODORO, A. J.; KOBLITZ, M. G. B. Estabilidade de ácido ascórbico em sucos de frutas frescos sob diferentes formas de armazenamento. Brazilian Journal Food and Technology, v. 17, n. 2, p. 139-145, 2014

DANTAS, R. L.; ROCHA, A. P. T.; ARAÚJO, A. S.; RODRIGUES, M. S. A.; MARANHÃO, T. K. L. Qualidade microbiológica de polpa de frutas comercializadas na cidade de Campina Grande. Revista Brasileira de Produtos Agroindustriais, v.14, n.2, p.125-130, 2012.

DEWANTO, V.; WU, X.; LIU, R.H. Processed sweet corn has higher antioxidant activity. Journal of Agricultural and Food Chemistry, v. 50, n. 17, p. 4959-4964, 2002

DEROSSI, A., DE PILLI, T.; FIORE, A.G. Vitamin C degradation of strawberry juice under non-isothermal conditions. Food Science and Technology, v.43, n.4, p.590-595, 2010.

FANTE, L. Estudo da cinética de branqueamento e de secagem por ar quente e liofilização do alho (Allium sativum L.). 2011. 119 f. Dissertação (Mestrado em Ciência e Tecnologia de alimentos) - Universidade Federal do Rio Grande do Sul - UFRS, 2011.

FERNÁNDEZ, A.; DOS SANTOS, M.; DA SILVA, D.; DE SOUSA, P.; MAIA, G. \& DE FIGUEIREDO, R. Chemical and physicochemical characteristics changes during passion fruit juice processing. Ciência e Tecnologia de Alimentos, v.31, n.3, p.747-751, 2011. 
GEORGÉ, S.; TOURNIAIRE, F.; GAUTIER, H.; GOUPY, P.; ROCK, E. \& CARIS, C. Changes in the contents of carotenoids, phenolic compounds and vitamin $\mathrm{C}$ during technical processing and lyophilisation of red and yellow tomatoes. Food Chemistry, v.124, n.4, p.1603$1611,2011$.

HAL, P.; BOSSCHAART, C.; TWISK, C.V.; VERKERK, R.; DEKKER, M. Kinetics of thermal degradation of vitamin $\mathrm{C}$ in marula fruit (Sclerocarya birrea subsp. caffra) as compared to other selected tropical fruits. LWT - Food Science and Technology, v.49, n.2, p.188-191, 2012.

HAMACEK, F. R.; SANTOS, P. R. G.; BEDETI, S. F.; CARDOSO, L. M.; RIBEIRO, S. M.; MARTINO, H. S.; MOREIRA, A. V. B.; SANT'ANA, H. M. P. Tamarindo do cerrado mineiro: caracterização física, físico-química, carotenóides e vitaminas. Nutrire, v.36, Sup, p.69-69, 2011.

MERCALI, G.; JAESCHKE, B.; TESSARO, I.; MARCZAK, L. Study of vitamin C degradation in acerola pulp during ohmic and conventional heat treatment. LWT - Food Science and Technology, v. 47, v.1, p. 91-95, 2012.

MATSUDA, H.; LLAVE, Y.; FUKUOKA, M.; SAKAI, N. Color changes in fish during grilling - Influences of heat transfer and heating medium on browning color. Journal of Food Engineering, v. 116, n.1, p. 130-137, 2013.

MOURA, S. C. S. R.; VITALI, A. A.; ALMEIRA, M.E. M.; BERBARI, S. A.; SIGRIST, J. M. M. Cinética de Degradação de Polpas de Morango. Brazilian Journal Food and Technology, v.4, p.115-121, 2001

NISHA, P.; SINGHAL, S.; PANDIT A. A study on degradation kinetics of ascorbic acid in amla (Phyllanthus emblica L.) during cooking. International Journal of Food Sciences and Nutrition, v. 55, n.5, p.415-422, 2004.

OLIVEIRA, A. N.; RAMOS, A. M.; CHAVES, J. P.; VALENTE, M. E. R. Cinética de degradação e vida-de-prateleira de suco integral de manga. Ciência Rural, Santa Maria, v.43, n.1, p.172-177, 2013.

ORDONEZ-SANTOS, L. E.; PORTILHA, M. A. P.; RODRIGUEZ, D. X. R. Cinética de degradação térmica de vitamina c em frutos de goiaba (Psidium guajava L.). Rev. Lasallista InvestigAción, v.10, n.2, p.44-51, 2013.

PATHARE, P.B.; OPARA, U.L.; AL-SAID, F.A-J. Colour measurement and analysis in fresh and processed foods. A review. Food and Bioprocess Technology, v.6, p. 36-60, 2013.

PATRAS, A.; BRUTON, N. P.; O'DONNELL, C.; TIWARI, B.K. Effect of thermal processing on anthocyanin stability in foods; mechanisms and kinetics of degradation. Trends in Food Science \& Technology, v. 21, n. 1, p. 3-11, 2010.

PAUL, R.; GHOSH, U. Effet or thermal treatment on ascorbic content of pomegranate juice. Indian Journal Biotechnology, v.11, p.309-312, 2012. 
PONKHAM, K.; MEESO, N.; SOPONRONNARIT, S.; SIRIAMORNPUN, S.. Modeling of combined far-infrared radiation and air drying of a ring shapedpineapple with/without shrinkage. Food and Bioproducts Processing. v.90, ed 2, p.155-164, 2012.

SANT'ANNA, V.; BRANDELLI, A.; MARCZAKA, L. D. F.; TESSARO, I. C. Kinetic modeling of total polyphenol extraction from grape marc and characterization of the extracts. Separation and Purification Technology, v. 100, p. 82-87, 2012.

SANTOS, J. S.; SANTOS, M. L. P.; AZEVEDO, A. S. Validação de um método para determinação simultânea de quatro ácidos orgânicos por cromatografia líquida de alta eficiência em polpas de frutas congeladas. Quimica Nova, v. 37, n. 3, p.540-544, 2014.

SPINOLA, V.; BERTA, B.; CÂMARA, J. S.; CASTILHO, P. C. Effect of Time and Temperature on Vitamin C Stability in Horticultural Extracts. UHPLC-PDA vs. Iodometric Titration as Analytical Methods. LWT - Food Science and Technology, v. 50, n. 2, p. 489495, 2013.

TARRAGO-TRANI, M. T.; PHILlIPS, K. M.; COTTY, M. Matrix-Specifi c Method Validation for Quantitative Analysis of Vitamin C in Diverse Foods. Journal of Food Composition and Analysis, v. 26, n. 1-2, p. 12-25, 2012.

TREVISAN, R.; TREPTOW, R. O.; GONÇALVES, E. D. Atributos de qualidade considerados pelo consumidor de Pelotas/RS, na compra de pêssego in natura. Revista Brasileira Agrociência, v. 12, n. 3, p. 371-374, 2006.

W U.; SUN, D. W. Colour measurements by computer vision for food quality control- a review. Trends in Food Science \& Technology, v.29, p. 5-20, 2013. 